\title{
Evidencia de una proteína de reserva vegetativa de 20 kDa en raíz de nogal (Carya illinoensis Koch) durante la etapa de letargo
}

\section{Evidence of a $20 \mathrm{kDa}$ vegetative storage protein in pecan (Carya illinoensis Koch) root during the dormancy stage}

\author{
Daniel Alejandro Espino-Castillo ${ }^{1}$, Luis Manuel Valenzuela-Núñez ${ }^{1 *}$, Juan Porfirio Legaria-Solano ${ }^{2}$, Edwin \\ Amir Briceño-Contreras ${ }^{1}$, Juan Ramón Esparza-Rivera ${ }^{3}$, Geremías Rodríguez-Bautista ${ }^{4}$, Cristina \\ García-de-la-Peña ${ }^{1}$ \\ ${ }^{1}$ Universidad Juárez del Estado de Durango. Facultad de Ciencias Biológicas. Av. Universidad s/n. CP. 35010 . Fracc. \\ Filadelfia, Gómez Palacio, Durango, México. \\ ${ }^{2}$ Universidad Autónoma Chapingo. Departamento de Fitotecnia. Carr. México-Texcoco Km 38.5. CP. 56230. Chapingo, \\ Texcoco, Edo. de Méx. \\ ${ }^{3}$ Universidad Juárez del Estado de Durango. Facultad de Ciencias Químicas Unidad Gómez Palacio. Av. Universidad s/n. CP. \\ 35010. Fracc. Filadelfia, Gómez Palacio, Durango, México. \\ ${ }^{4}$ Colegio de Postgraduados. Posgrado en Recursos Genéticos y Productividad-Genética. Campus Montecillo, Km 36.5 Carr. \\ México-Texcoco. CP. 56230, Texcoco, Edo. de México. México \\ *Autor de correspondencia: luisvn70@hotmail.com
}

Nota científica recibida: 22 de agosto de 2017 aceptada: 25 de enero de 2018

RESUMEN. Se realizó un análisis de proteínas totales solubles y de proteínas de reservas vegetativas (PRV), en tejidos de árboles de nogal (Carya illinonensis Koch var. Western) en letargo y producción. La determinación de proteínas totales solubles se realizó por el método de Bradford, por medio de electroforesis en gel de poliacrilamida al 15\% para detectar las PRV presentes en los tejidos vegetales. Los resultados indican que las ramas y los brotes de crecimiento anual fueron los órganos con mayor concentración de proteínas totales solubles en letargo, mientras que en producción no se presentó diferencia significativa de concentración entre los diferentes tejidos estudiados. Se detectó un polipéptido de $20 \mathrm{kDa}$ con características de una PRV en la raíz en la etapa de letargo.

Palabras clave: Órganos perennes, frutales, fenología, proteínas, PRV

ABSTRACT. An analysis of total soluble proteins and vegetative storage proteins (VSPs) in the tissues of pecan (Carya illinonensis Koch var. Western) trees in dormancy and production was carried out. Total soluble proteins were determined using the Bradford method, by means of electrophoresis in 15\% polyacrylamide gel, to detect the VSPs present in the plant tissues. The results indicate that the branches and shoots of annual growth were the organs with the highest concentration of total soluble proteins in dormancy, while in production there was no significant difference in concentration between the different tissues studied. A 20-kDa polypeptide with characteristics of a VSP was detected in the root in the dormancy stage.

Key words: Perennial organs, fruit trees, phenology, proteins, VSP

\section{INTRODUCCIÓN}

Las primeras plantaciones de nogal en la Comarca Lagunera se establecieron en 1948 (Potisek et al. 2010), actualmente el valor de la producción de nuez ocupa el primer lugar entre los frutales de la región, con valor aproximado de 200 millones de pesos (García et al. 2009); superficie cosechada de 5534 ha y producción de 7600 toneladas (SIAP 2015). El nitrógeno (N) es el elemento de mayor demanda en las plantas, constituye un factor limitante para su desarrollo y crecimiento, siendo esencial para aumentar la producción y mejorar la calidad. Este macro nutriente altera la composición de las plan- 
tas, pudiendo modificar el contenido de azúcares, proteínas y lípidos (Okumoto y Pilot 2011, Sławomir 2017). La mayor parte del $\mathrm{N}$ en el suelo se asimila en forma de compuestos orgánicos como proteínas, lignina o quitina, que no pueden ser directamente utilizadas por las plantas, sino que requieren un proceso de polimerización por parte de microorganismos especializados, para convertirse en $\mathrm{N}$ orgánico o mineral (Farrell et al. 2014, Liu 2016). El N es abundante en las plantas, y dentro de las formas de nitrógeno orgánico se encuentran las proteínas solubles, siendo esencial este macro nutriente para el establecimiento de nuevas estructuras celulares, metabólicas y de codificación genética; mientras que su falta y/o deficiencia produce disminución del crecimiento (Tan-Wilson y Wilson 2012, Sławomir 2017).

Trabajos recientes enfocados en la fenología de los árboles a lo largo de un ciclo, consideran que los procesos fisiológicos no son regulados solo por la temperatura (Wildhagen et al. 2010, Heide 2011, Rohde et al. 2011). Para un mejor conocimiento del comportamiento fenológico de los árboles, debe tomarse en cuenta que el nitrógeno estacional es afectado por la temperatura (Wildhagen et al. 2013). Mientras que la asimilación del nitrógeno por las raíces se regula por su disponibilidad en el suelo y la demanda para el crecimiento y desarrollo del árbol completo (Millard 1996). Entre los factores de demanda de nitrógeno para el desarrollo y crecimiento se encuentra la fructificación (Rennenberg y Geßler 1999), el desarrollo foliar y radicular (Silla y Escudero 2003), y la senescencia (Kunkle et al. 2009). Además de factores externos, como la salinidad (Gimeno et al. 2009), el calor, la sequía (Rennenberg et al. 2006), el nitrógeno atmosférico y la concentración de ozono (Nair y Padmavathy 2014, Rashied et al. 2015, Hewittac et al. 2016, Rebouças et al. 2017), factores que son de particular importancia para el desarrollo del nogal en la región de estudio.

Las proteínas solubles son uno de los principales componentes orgánicos del protoplasma, las cuales son moléculas complejas que contienen nitrógeno, diferentes unas de otras por su forma, tamaño, propiedades de superficie y función (Miao y Jiang 2007). Tienen en común que se forman a partir de los aminoácidos, tienen propiedades coloidales y son anfóteras (Yokoyama et al. 2016). Una parte de estas proteínas insolubles se integran en diferentes estructuras celulares, principalmente en las membranas y paredes de los diferentes compartimentos celulares. De todas las proteínas de las células vivas, alrededor del 30\% corresponde a este tipo de proteínas estructurales (Sauter y Wellenkamp 1998, Pasiakos et al. 2015); mientras que el resto se encuentran como proteínas solubles, las cuales juegan un papel esencial en las secuencias metabólicas y en los órganos perennes, debido a que realizan funciones de almacenamiento de nitrógeno (Valenzuela et al. 2010, Valenzuela et al. 2011, Wan-Ze et al. 2012).

Las proteínas de reserva vegetativas (PRV) son depósitos de nitrógeno importantes en muchas plantas, estas fueron descritas por primera vez en semillas, por su papel en el suministro de nitrógeno a las plántulas al iniciar el crecimiento (Staswick 1994). Se acumulan en grandes cantidades en hojas, tallos tubérculos y raíces (Avice et al. 2011, Xue et al. 2016). En muchas especies leñosas se localizan principalmente en el parénquima del xilema y floema, almacenándose en mayor proporción durante el otoño e invierno en órganos perennes y luego desaparecen cuando se reanuda el crecimiento en la primavera (Valenzuela et al. 2010, Valenzuela et al. 2011, El Zein et al. 2011). En los árboles caducifolios, durante la etapa de letargo, las raíces y los tallos alcanzan el máximo nivel de almacenamiento de reservas, las cuales disminuyen en la brotación de yemas, y en las primeras etapas de crecimiento de brotes y hojas (Martínez et al. 2013). La concentración de reservas disminuye durante el desarrollo vegetativo, se presenta una cantidad máxima de éstas al final del verano y principios de otoño (El Zein et al. 2011, Valenzuela et al. 2011, Valenzuela et al. 2014). Dadas las características de frutal caducifolio y la escasez de estudios relacionados con la fisiología de frutales caducifolios, el objetivo del estudio fue analizar el comportamiento de las proteínas totales solubles y PRV en las etapas fenológicas 
de letargo y producción, de órganos perennes de nogal Carya illinoensis Koch.

\section{MATERIALES Y MÉTODOS}

\section{Área de estudio}

El estudio se llevó a cabo en la huerta experimental de la Universidad Autónoma Agraria Antonio Narro Unidad Laguna en Torreón, Coahuila, ubicada a $25^{\circ} 33^{\prime} 22.63^{\prime \prime}$ LN y $103^{\circ} 22^{\prime} 07.77^{\prime \prime}$ LO. Se encuentra bajo manejo agronómico con riego por inundación con agua de pozo, la edad de los árboles en promedio es de 40 años, la variedad establecida es Western, la densidad de plantación es de 100 árboles ha ${ }^{-1}$ establecidos en marco real. El calendario incluye ocho riegos con intervalos de 12 a $47 \mathrm{~d}$, dependiendo de la etapa fenológica con lámina de riego total de $748 \mathrm{~mm}$ al año. $\mathrm{El} \mathrm{pH}$ del agua es de 8.2 y la conductividad eléctrica de 1480 microohmios $\mathrm{cm}^{-1}$. El clima de la región es seco desértico con precipitación media anual de $230 \mathrm{~mm}$ (IMTA 2013) y altitud de 1120 m (INEGI 2012).

\section{Muestreo}

El muestreo se realizó de forma sistematizada, seleccionando los árboles y muestras al azar. Para ello, se eligieron cuatro árboles adultos de forma intercalada en la parte media de la parcela para evitar el efecto de borde. En cada árbol se tomaron dos muestras de cada parte muestreada, de la raíz principal se tomó a $40 \mathrm{~cm}$ de profundidad, del tronco a una altura de $1.30 \mathrm{~m}$, de la rama se tomaron a un metro de la parte donde se diferencian las ramas, y del crecimiento anual las muestras se tomaron del brote emitido por el árbol el año en que se realizó el estudio. Los muestreos se realizaron en las etapas fenológicas de producción (cuando el árbol se encuentra en fructificación y presenta el máximo crecimiento vegetativo, a los 60 DDF (días después de floración)) y en letargo (cuando el árbol se encuentra defoliado sin crecimiento vegetativo aéreo, a los 210 DDF). Las muestras de tronco y ramas se tomaron con un taladro Pressler en forma de virutas, mientras que las muestras de crecimien- tos anuales se obtuvieron con la ayuda de un serrucho convencional y las muestras de raíz se obtuvieron por extracción con la ayuda de un serrucho convencional, realizando una zanja a $40 \mathrm{~cm}$ de profundidad para localizar la raíz principal.

Las muestras se limpiaron y se colocaron en bolsas de aluminio perforadas, que se etiquetaron y almacenarse en una hielera, para luego congelarlas en nitrógeno líquido y almacenarlas en un ultracongelador (Revco Value Plus ${ }^{\circledR}$ ThermoScientific ${ }^{\circledR}$ ) a $-70{ }^{\circ} \mathrm{C}$ para detener todos los procesos bioquímicos en los tejidos, posteriormente se liofilizaron en un liofilizador (Labconco Freezone Triad ${ }^{\circledR}$ Freeze Dry Systems ${ }^{\circledR}$ ) durante siete días a $-40{ }^{\circ} \mathrm{C}$; para deshidratar las muestras por sublimación. Las muestras liofilizadas se pulverizaron con un molino de cuchillas (Fritsch ${ }^{\circledR}$ Pulverisette $15^{\circledR}$ ), para obtener un polvo fino. Para luego tomar $10 \mathrm{mg}$ de materia seca en microtubos (MCT-200-C Clear Axygen Scientific ${ }^{\mathbb{R}}$ ).

\section{Determinación de proteínas totales solubles}

La extracción y cuantificación de proteínas se realizó por el método de Bradford (1976). Para lo cual se preparó una solución de extracción (0.1 $\mathrm{M} \mathrm{KH}_{2} \mathrm{PO}_{4}, 0.1 \mathrm{M} \mathrm{Na}_{2} \mathrm{HPO}_{4} 5 \mathrm{mM}$ DTT, $0.3 \%$ (m/v) PEG y $13 \mathrm{mg}$ PVP). De la que se agregó 1 $\mathrm{mL}$ a los microtubos que contenían los $10 \mathrm{mg}$ de materia seca, para luego colocar un balín de acero a cada uno de los microtubos y agitar en un vortex (Maxi Mix II ${ }^{\circledR}$ Thermo Scientific ${ }^{\circledR}$ ) durante 3 min para romper las paredes celulares, posteriormente los microtubos se centrifugaron en una centrífuga Spectrafuge $16 \mathrm{M}{ }^{\circledR}$ Labnet ${ }^{\circledR}$ a $10000 \mathrm{rpm}$ a $4{ }^{\circ} \mathrm{C}$ durante $15 \mathrm{~min}$, para luego extraer $500 \mu \mathrm{L}$ de cada microtubo y colocarlos en celdillas de lectura y agregar $500 \mu \mathrm{L}$ de la solución Quickstart ${ }^{\circledR}$ Bradford ${ }^{\circledR}$, agitar y dejar reposar por $5 \mathrm{~min}$ a temperatura ambiente. La lectura se realizó a absorbancia de 595 $\mathrm{nm}$ en un espectrofotómetro UV-Visible (Genesys $20^{\circledR}$ Thermo Scientific ${ }^{\circledR}$ ). La curva de calibración para determinar la concentración de proteínas totales solubles se realizó con Bovine Serum Albumine (BSA) como estándar con una $\mathrm{R}^{2}$ de 0.9912 . 


\begin{tabular}{|c|c|c|c|c|}
\hline & Raíz & Tronco & Rama & CA \\
\hline Media & 32.52 & 28.58 & 31.27 & 32.93 \\
\hline Error típico de la media & 1.70 & 1.45 & 4.62 & 3.00 \\
\hline Desviación típica & 6.80 & 5.80 & 18.48 & 12.00 \\
\hline U de Mann-Whitney & 11.000 & 21.000 & 16.000 & 16.000 \\
\hline $\mathrm{p}$ & 0.027 & 0.248 & 0.093 & 0.092 \\
\hline
\end{tabular}

\section{Electroforesis en gel de poliacrilamida}

Las proteínas presentes en los extractos se usaron para llevar a cabo la electroforesis en gel de poliacrilamida al $15 \%$. Las soluciones se desnaturalizaron a temperatura de $50{ }^{\circ} \mathrm{C}$ por $3 \mathrm{~min}$, centrifugaron a $12000 \mathrm{rpm}$ durante $5 \mathrm{~min}$ y añadieron $40 \mu \mathrm{L}$ de cada una de las soluciones al gel de acrilamida dentro de la cámara de electroforesis con solución amortiguadora de corrida: Tris base 0.025 M, glicina 0.192 M y SDS al 0.1\% (Laemmli 1970). La electroforesis se realizó aplicando una corriente de $120 \mathrm{~V}$, durante $4 \mathrm{~h}$, cuando el frente del colorante (azul de bromofenol) llegó al margen inferior del gel se suspendió la electroforesis. Después de retirar el gel de la cámara de electroforesis, se sumergió por un día en solución azul de Coomasiie al $0.1 \%$, metanol al $50 \%$ y ácido acético al $10 \%$. Al día siguiente se decoloró con una solución de metanol al 5\% y ácido acético glacial al $7 \%$ para eliminar el fondo azul del gel y visualizar los polipéptidos coloreados en un transluminador de luz blanca.

\section{Análisis estadístico}

Se realizó la prueba de Mann-Whitney para la comparación de medias entre etapas fenológicas y la prueba de Kruskal-Wallis ( $\mathrm{p}<0.05$ ) para la comparación de medias entre órganos perennes. Todos los análisis se realizaron con el software SPSS Versión 18.

\section{RESULTADOS Y DISCUSIÓN}

En la etapa de producción, las ramas presentaron la mayor concentración de proteínas (31.23 $\mathrm{mg} \mathrm{g}^{-1}$ de MS) seguida de la raíz $\left(28.78 \mathrm{mg} \mathrm{g}^{-1}\right.$ de
MS), el crecimiento anual (26.58 $\mathrm{mg} \mathrm{g}^{-1}$ de MS) y el tronco (26.24 $\mathrm{mg} \mathrm{g}^{-1}$ de MS) (Tabla 1). En la etapa de letargo, el crecimiento anual tuvo la mayor concentración (39.27 $\mathrm{mg} \mathrm{g}^{-1}$ de MS), seguido de la raíz (36.26 $\mathrm{mg} \mathrm{g}^{-1}$ de MS), la rama (31.23 $\mathrm{mg} \mathrm{g}^{-1}$ de MS) y el tronco (30.91 $\mathrm{mg} \mathrm{g}^{-1}$ de MS). Estos resultados sugieren una acumulación preferencial en las partes aéreas, lo que es un comportamiento inverso a lo observado por Martínez et al. (2013), debido a que estas reservas permanecen en los tejidos aéreos con la finalidad de ser utilizadas para la respiración durante el invierno (El Zein et al. 2011, Valenzuela et al. 2011, Valenzuela et al. 2014).

Para la PRV en geles SDS, se detectó un polipéptido de $20 \mathrm{kDa}$ en la etapa de letargo (Figura $1)$, que se presenta en una cantidad importante con respecto a los otros polipéptidos, que está ausente en el crecimiento vegetativo y en letargo. De acuerdo con la definición de Chapin et al. (1990) el polipétido detectado corresponde a una PRV, por el valor de $20 \mathrm{kDa}$ del polipéctido se encuentra dentro de los valores reportados para la PRV de otras especies de árboles, con valores en masa molecular que varía entre 15 y 45 kDa (Gómez y Faurobert 2002, Valenzuela et al. 2011, El Zein et al. 2011, Tian et al. 2011, Pettengill et al. 2013).

Los resultados del análisis de las proteínas de los diferentes órganos entre las etapas fenológicas de producción y letargo muestran diferencias significativas en la concentración de proteínas en la raíz $(U=11.000, P=0.027)$, pero no se detectaron diferencias significativas entre los órganos (Tabla 1 y Figura 2). Estos resultados coinciden con los reportados para especies caducifolias por Martínez et al. (2013), El Zein et al. (2011), Valenzuela et al. 


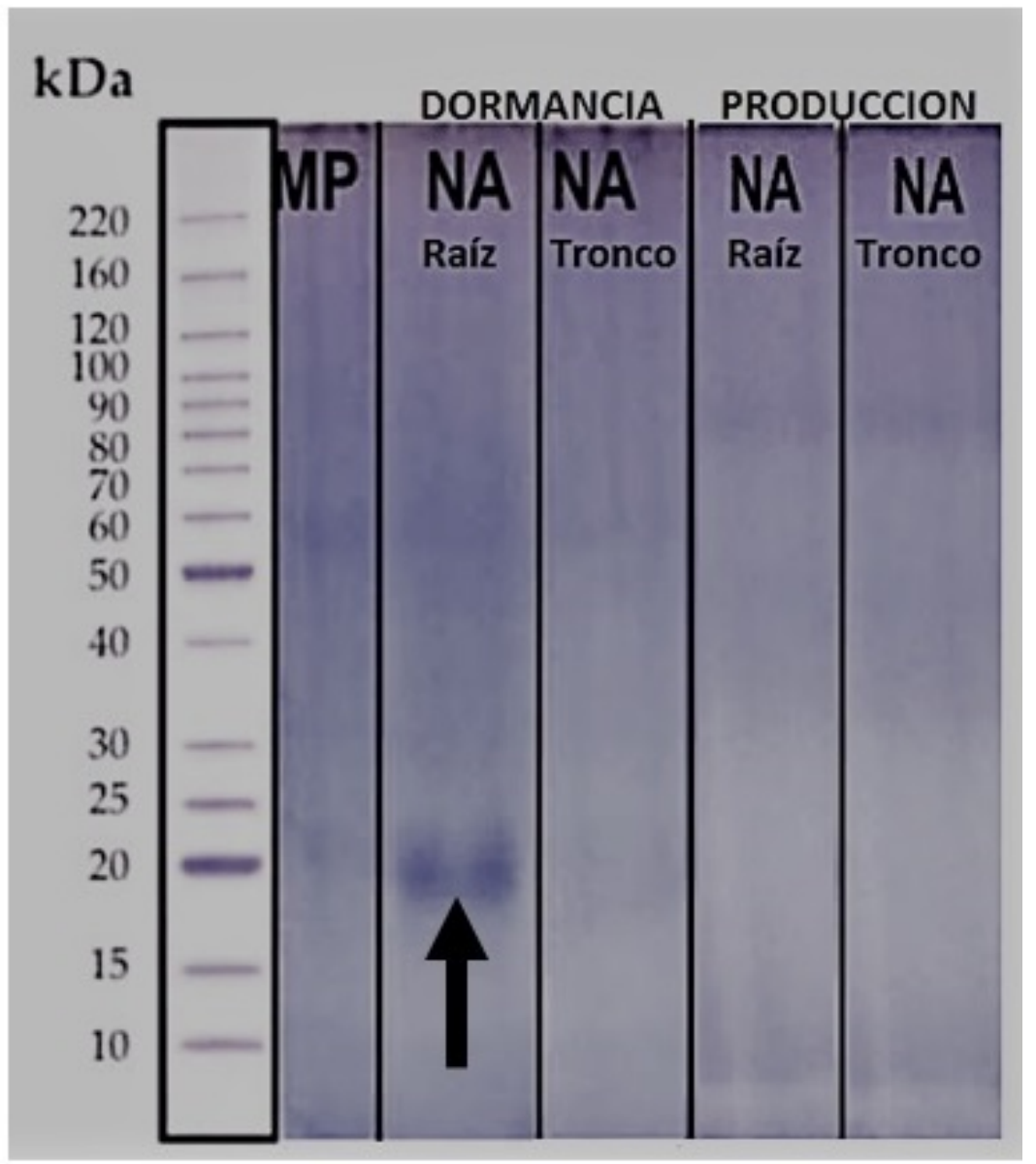

Figura 1. Perfil de electroforesis en gel de poliacrilamida de proteínas en tejidos de raíz y tronco de árboles de nogal (Carya illinoensis) (letargo y producción). $\mathrm{NA}=$ Muestra tomada, $\mathrm{MP}=$ Marcador.

(2011) y Valenzuela et al. (2014). Durante el invierno, se activa el crecimiento en los tejidos de la raíz en los árboles deciduos, al mismo tiempo que el crecimiento de la biomasa aérea (tronco, ramas) se detiene; mientras que en verano se presenta este comportamiento de manera inversa (Valenzuela et al. 2010). Se encontró que la raíz en la etapa de letargo fue el órgano vegetal que presentó la mayor concentración de proteínas. Al respecto, Barbaroux et al. (2003) menciona que las reservas se concentran y tienen mayor demanda en los crecimientos anuales, lo que favorece el desarrollo vegetativo de la planta, lo que se observó en el presente estudio.

\section{AGRADECIMIENTOS}

Al Dr. Angel Lagarda Murrieta por la disponibilidad para la realización del estudio en la huerta experimental de la Universidad Autónoma Agraria Antonio Narro Unidad Laguna, a la M.C. Carmen Potisek Talavera por su apoyo en el muestreo, al personal del Laboratorio de Genética del Departamento de Fitotecnia de la Universidad Autónoma Chapingo por la disponibilidad con el equipo transluminador para geles y al Consejo Nacional de Ciencia y Tecnología por el apoyo para la realización de los estudios de maestría de Edwin A. Briceño Contreras y Daniel A. Espino Castillo. 


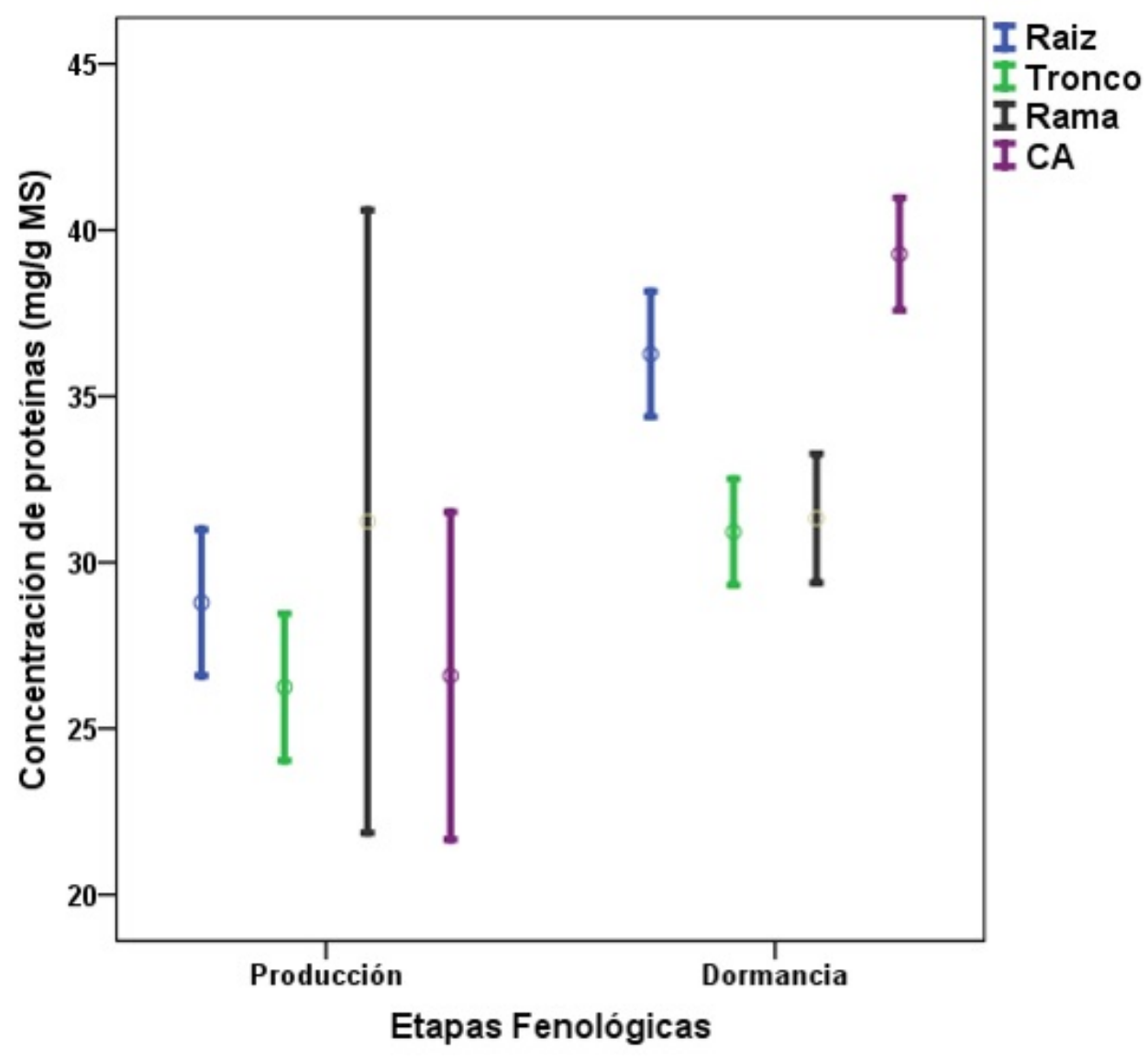

Figura 2. Media y error estándar del peso en $\mathrm{mg}$ proteínas $\mathrm{g}^{-1} \mathrm{MS}$ de raíz, tronco, rama y crecimiento anual (CA) en dos etapas fenológicas.

\section{LITERATURA CITADA}

Avice JC, Dily F, Goulas E, Noquet C, Meuriot F, Volenec JM, et al. (2011) Vegetative storage proteins in overwintering storage organs of forage legumes: Roles and regulation. Canadian Journal of Botany 81: 1198-1212

Bradford MM (1976) A rapid and sensitive method for the quantification of microgram quantities of protein utilizing the principle of protein dry binding. Analytical Biochemistry 72: 248-254

Barbaroux C, Bréda N, et Dufrêne E (2003) Distribution of above-ground and below-ground carbohydrate reserves in adult trees of two contrasting broad-leaved species (Quercus petraea and Fagus sylvatica). New Phytologist 157: 605-615

Chapin FS, Schulze ED, Mooney HA (1990) The ecology and economics of storage in plants. Annual Review of Ecology and Systematics 21: 423-447

El Zein R, Maillard P, Bréda N, Marchand J, Montpied P, Gérant D (2011) Seasonal changes of C and N non-structural compounds in the stem sapwood of adult sessile oak and beech trees. Tree Physiology 31: 843-854. 
Farrell M, Prendergast-Miller M, Davey L, Jones DL, Hill PW, Condron LM (2014) Soil microbial organic nitrogen uptake is regulated by carbon availability. Soil Biology \& Biochemistry 77: 261-267

García-Hernández JL, Orona-Castillo I, González-Cervantes G, Valdez-Cepeda R, Murillo-Amador B, TroyoDiéguez $\mathrm{E}$, et al. (2009) Interacciones nutrimentales y normas de diagnóstico de nutrimento compuesto en nogal pecanero (Carya illinoensis). Revista Chapingo Serie Horticultura 15(2): 141-147.

Gimeno V, Syvertsen JP, Nieves M, Simon I, Martinez V, Garcia-Sanchez F (2009) Additional nitrogen fertilization affects salt tolerance of lemon trees on different rootstocks. Scientia Horticulturae 121: 298-305.

Gomez L, Faurobert M (2002) Contribution of vegetative storage proteins to seasonal nitrogen variations in the young shoots of peach trees (Prunus persica L. Batsch). Journal of Experimental Botany 53: 2431-2439.

Hänninen H, Tanino K (2011) Tree seasonality in a warming climate. Trends in Plant Science 16: 412-416.

Heide OM (2011) Temperature rather than photoperiod controls growth cessation and dormancy in Sorbus species. Journal of Experimental Botany 62: 5397-5404.

Hewittac DKL, Millsa G, Hayesa F, Norrisa D, Coyleb M, Wilkinsonc S, et al (2016) N-fixation in legumes An assessment of the potential threat posed by ozone pollution. Environmental Pollution 208: 909-918

IMTA (2013) Extractor rápido de información climatológica. Versión 3.2. (ERIC 3.2). IMTA. Secretaría del Medio Ambiente y Recursos Naturales. México. Software en CD.

INEGI (2012) Anuario estadístico del estado de Coahuila de Zaragoza. Instituto Nacional de Estadística y Geografía. Aguascalientes, Ags. México. 501p.

Kunkle JM, Walters MB, Kobe RK (2009) Senescence related changes in nitrogen in fine roots: mass loss affects estimation. Tree Physiology 29: 715-723.

Laemmli UK (1970) Cleavage of structural proteins during the assembly of the head of bacteriophage T4. Nature 227: 680-685.

Liu, Qiao N, Xu X, Xin X, Han JY, Tian Y, et al (2016) Nitrogen acquisition by plants and microorganisms in a temperate grassland. Scientific Reports 6: 22642 doi:10.1038/srep22642

Martínez T, Plascencia F, Islas L (2013) La relación entre los carbohidratos y la vitalidad en árboles urbanos. Red de Revistas Científicas de América Latina, el Caribe, España y Portugal. Revista Chapingo Serie Ciencias Forestales y del Ambiente 19: 459-468.

Miao Y, Jiang L (2007) Transient expression of fluorescent fusion proteins in protoplasts of suspension cultured cells. Nature Protocols 2: 2348-53

Millard P (1996) Ecophysiology of internal cycling of nitrogen for tree growth. Zeitschrift fu“ $r$ Pflanzenerna"hrung \& Bodenkunde 159: 1-10.

Nair DN, Padmavathy S (2014) Impact of endophytic microorganisms on plants, environment and humans. The Scientific World Journa. Article ID 250693.

Okumoto S, Pilot G (2011) Amino acid export in plants: A missing link in nitrogen cycling. Molecular Plant 4: 453-463.

Pasiakos SM, Agarwal S, Lieberman HR, Fulgoni VL (2015) Sources and amounts of animal, dairy, and plant protein intake of US adults in 2007-2010. Nutrients 7: 7058-7069. 
Pettengill EA, Pettengill JB, Coleman GD (2013) Elucidating the evolutionary history and expression patterns of nucleoside phosphorylase paralogs (vegetative storage proteins) in Populus and the plant kingdom. BMC Plant Biology13:118. Doi: 10.1186/1471-2229-13-118.

Potisek MC, González G, Valenzuela L, González JJ, Velásquez M (2010) Respuesta del desarrollo y calidad de plantas de nogal pecanero a la fertilización nitrogenada bajo condiciones de invernadero. Agrofaz 10 (3): 191-197

Rashied T, MasahiroY, Yoshiharu W, Ryo F, Takeshi I (2015) Effects of ozone on growth, net photosynthesis and yield of two African varieties of Vigna unguiculata. Environmental Pollution 196: 230-238.

Rebouças DM, De Sousa YM, Bagard M, Costa JH, Jolivet Y, Fernandes De Melo D, et al. (2017) Combined effects of ozone and drought on the physiology and membrane lipids of two cowpea (Vigna unguiculata (L.) Walp) cultivars. Plants 6: 14. Doi:10.3390/plants6010014

Rennenberg H, Geßler A (1999) Consequences of N deposition to forest ecosystems - recent results and future research needs. Water, Air and Soil Pollution 116: 47-64.

Rennenberg H, Loreto F, Polle A, Brilli F, Fares S, Beniwal RS, Gessler A (2006) Physiological responses of forest trees to heat and drought. Plant Biology 8: 556-571.

Rohde A, Bastien C, Boerjan W (2011) Temperature signals contribute to the timing of photoperiodic growth cessation and bud set in poplar. Tree Physiology 31: 472-482.

Sauter JJ, Wellenkamp S (1998) Seasonal changes in content of starch, protein and sugars in the twig wood of Salix caprea L. Holzforschung 52: 255-262

SIAP (2015) Cierre de la producción agrícola por cultivo en ciclos perennes 2015 modalidad riego en la Región Lagunera: Coahuila-Durango, México. http://infosiap.siap.gob.mx/aagricola_siap_gb/ientidad/index.jsp. Fecha de consulta: 10 de abril de 2017.

Silla F, Escudero A (2003) Uptake, demand and internal cycling of nitrogen in saplings of Mediterranean Quercus species. Oecologia 136: 28-36.

Sławomir B, Marzena-Kalemba E, Pukacka S, Pietrowska-Borek M, Stawiński S, Ratajczak L (2017) Nitrate simultaneously enhances lipid and protein accumulation in developing yellow lupin cotyledons cultured in vitro, but not under field conditions. Journal of Plant Physiology 216: 26-34.

Staswick PE (1994) Storage proteins of vegetative plant tissue. Annual Review of Plant Physiology and Plant Molecular Biology 45: 303-322.

Tan-Wilson AL, Wilson KA (2012) Mobilization of seed protein reserves. Physiologia Plantarum 145:140-53

Valenzuela LM, Gérant D, Maillard P, Bréda NJJ (2010) Intra-tree distribution and seasonal dynamics of total soluble proteins in adult sessile oak (Quercus petraea L.) and common beech (Fagus sylvatica L.). Revista Mexicana de Ciencias Forestales 1: 105 - 117

Valenzuela LM, Gérant D, Maillard P, Bréda N, Gonzalez G, Sanchez I (2011) Evidence for a 26kDa vegetative storage protein in the stem sapwood of mature pedunculate oak. Interciencia 36: 142-147.

Valenzuela LM, Maillard P, González JL, González G (2014) Balance de carbohidratos en diferentes compartimentos vegetales de encino (Quercus petraea) y haya (Fagus sylvatica), sometidos a defoliación y sombra. Revista Chapingo Serie Zonas Aridas 13: 33-38.

Wan-Ze Z, Min C, San-Gen W, Wen-Fan X, Mai-He L (2012) Seasonal dynamics of mobile carbon supply in Quercus aquifolioides at the upper elevational limit. PLoS One 7: e34213. 
Wei-Min T, Ji-Lin W, Bing-Zhong H, Zheng-Hai H (2011) Vegetative storage proteins in the tropical tree Swietenia macrophylla: Seasonal fluctuation in relation to a fundamental role in the regulation of tree growth. Canadian Journal of Botany 81: 492-500.

Wildhagen H, Durr J, Ehlting B, Rennenberg H (2010) Seasonal nitrogen cycling in the bark of fieldgrown Grey poplar is correlated to meteorological factors and gene expression of bark storage proteins. Tree Physiology 30: 1096-1110.

Wildhagen H, Bilela S, Rennenberg H (2013) Low temperatures counteract short-day induced nitrogen storage, but not accumulation of bark storage protein transcripts in bark of grey poplar (Populus $\mathrm{X}$ canescens) trees. Plant Biology 15: 44-56.

Xue L, Frost C, Tsai CJ, Harding S (2016) Drought response transcriptomes are altered in poplar with reduced tonoplast sucrose transporter expression. Scientific Reports 6: 33655. Doi: 10.1038/srep33655

Yokoyama R, Kuki H, Kuroha T, Nishitani K (2016) Arabidopsis regenerating protoplast: a powerful model system for combining the proteomics of cell wall proteins and the visualization of cell wall dynamics. Proteomes 17(4). Doi: 10.3390/proteomes4040034 
